\title{
Resolving the Mechanisms of Soy Glycinin Self-Coacervation and Hollow-Condensate Formation
}

\author{
Nannan Chen,* Ziliang Zhao, Yong Wang, and Rumiana Dimova* \\ Cite This: ACS Macro Lett. 2020, 9, 1844-1852 \\ Read Online
}

ABSTRACT: Self-coacervation of animal-derived proteins has been extensively investigated while that of plant proteins remains largely unexplored. Here, we study the process of soy glycinin self-coacervation and transformation into hollow condensates. The protein hexameric structure composed of hydrophilic and hydrophobic polypeptides is crucial for coacervation. The process is driven by charge screening of the intrinsically disordered region of acidic polypeptides, allowing for weak hydrophobic interactions between exposed hydrophobic polypeptides. We find that the coacervate surface exhibits order, which stabilizes the coacervate shape during hollow-condensate formation. The latter process occurs via nucleation and growth of protein-poor phase in the coacervate interior, during which another ordered layer at the inner surface is

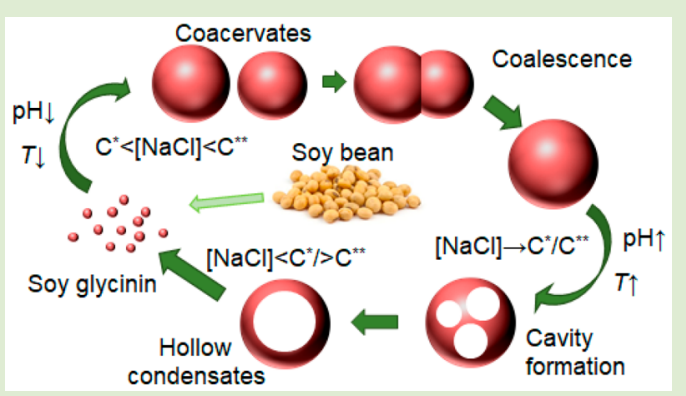
formed. Aging enhances the stability of both coacervates and hollow condensates. Understanding plant protein coacervation holds promises for fabricating novel functional materials.

$\mathrm{P}$ rotein coacervation or liquid-liquid phase separation in protein solutions is characterized by the formation of liquid-like protein-rich microdroplets (coacervates or condensates). Upon coalescence, they yield a macroscopically monophasic biomacromolecular fluid of highly concentrated protein phase. ${ }^{1}$ Coacervation can occur spontaneously upon changing the environmental conditions, a process known as simple coacervation or self-coacervation, ${ }^{2}$ or via interactions with another oppositely charged protein specie, also known as complex coacervation. ${ }^{3}$ Protein coacervation represents a crucial route for the assembly of cellular organelles ${ }^{4,5}$ and, at the same time, offers a facile approach toward the fabrication of biomaterials such as fibers, ${ }^{6}$ bioadhesives, ${ }^{7}$ and bioactive compound carriers. ${ }^{8}$ In recent years, much interest has been dedicated to human intracellular condensates originating from intrinsically disordered proteins as well as coacervates that derive from animal extracellular matrix proteins. ${ }^{4-7}$ Far less studies have focused on plant protein coacervation.

Compared to animal proteins, plant, and in particular soy proteins are widely abundant and economical, which makes them ideal materials for the scale-up processing in industry. Soy glycinin is one of the major storage proteins in the soybean seed. It is commonly used as an emulsion stabilizer for improving the texture of food products via modulating the structure of protein gels and for producing bioadhesives. ${ }^{9-11}$ Recently, soy glycinin was found to undergo self-coacervation in aqueous solutions upon the addition of salt, a process exemplified by the formation of spherical condensates in the micrometer range. ${ }^{12}$ Interestingly, upon a temperature increase, the coacervates transform into stable hollow microcapsule-like condensates (also referred to as vacuolated structures or a vesicle-like condensate phase). ${ }^{12,13}$ This process represents a simple and very energy-efficient route for microencapsulation of active compounds for controlled release, protection against environmental factors, or masking of an unpleasant odor or taste. ${ }^{14-16}$ By forming a protein layer on oil droplets, protein self-coacervation can be employed for the encapsulation of hydrophobic compounds, ${ }^{17}$ which represent a much simpler approach compared to traditional methods based on complex coacervation with another polymer, usually polysaccharide. ${ }^{14}$ Hollow protein microcapsules formed via coacervation can encapsulate and control the release of hydrophilic compounds by modulating the permeability of the protein shell. ${ }^{13,18}$ This is advantageous compared to more popular methods for microcapsule production such as the template-based layer-by-layer method, ${ }^{19}$ which involves a tedious and time-consuming fabrication process and is associated with waste of materials.

Despite the above-mentioned advantages and potential applications, the mechanism of soy glycinin self-coacervation and the cavitation of the condensates is not yet understood. Remarkably, the formation of hollow condensates upon selfcoacervation has not yet been reported for animal proteins, while it seems a common phenomenon for the plant seed $11 \mathrm{~S}$

Received: October 1, 2020

Accepted: November 30, 2020

Published: December 6, 2020 

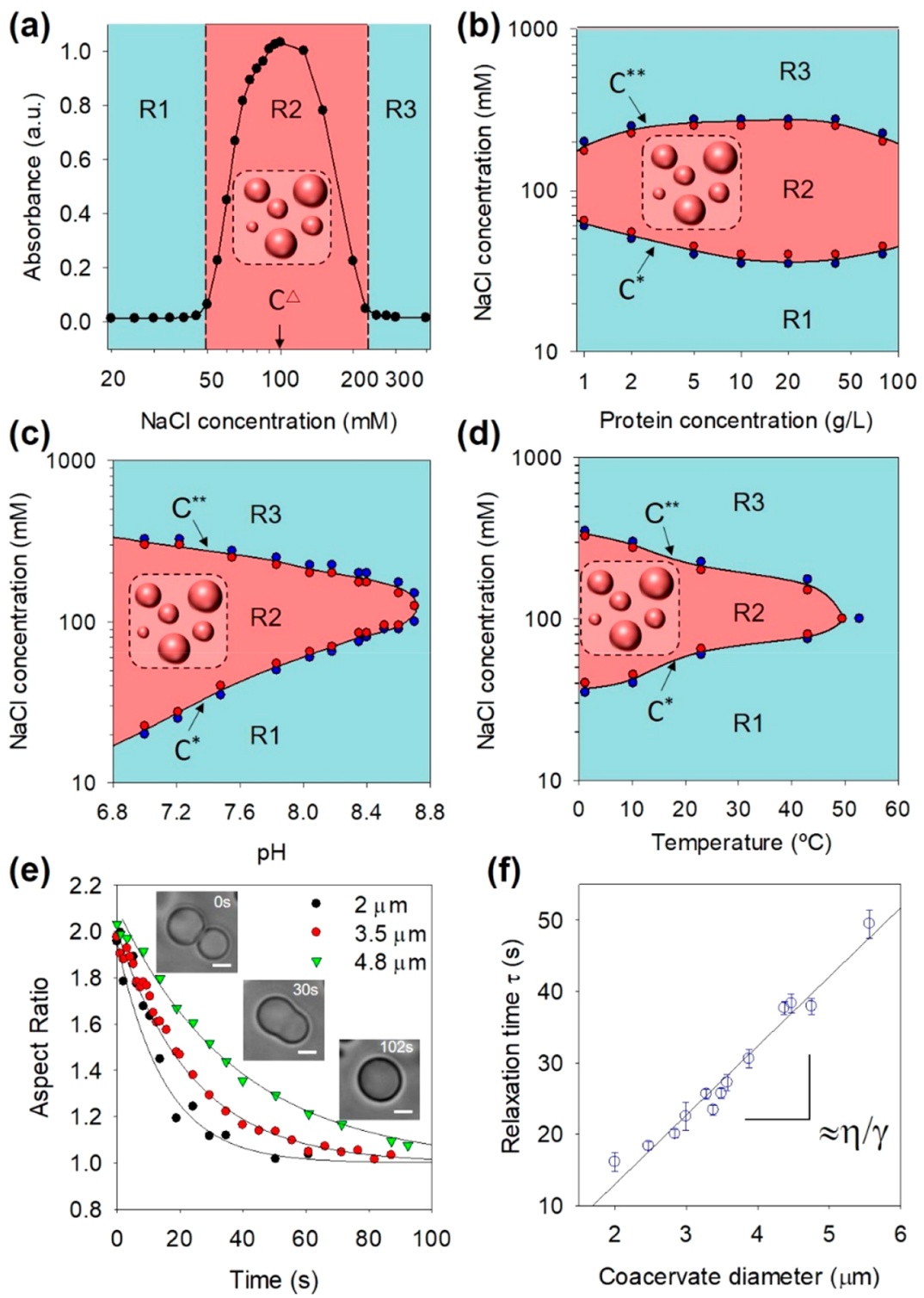

Figure 1. Phase diagrams of soy glycinin coacervation $(a-d)$ and physicochemical characterization of the condensates (e, f). (a) Protein solution $(11 \mathrm{~g} / \mathrm{L}, \mathrm{pH} 7.8)$ was adjusted to different ionic strengths at $23^{\circ} \mathrm{C}$ by adding $\mathrm{NaCl}$ solution with different concentrations, attaining a final protein concentration of $10 \mathrm{~g} / \mathrm{L}$. Turbidity of soy glycinin suspensions at different salt concentration are given; the framed cartoons (also in panels b-d) illustrate the emergence of coacervates in the respective phase region. (b) Phase state of soy glycinin as a function of protein concentration at room temperature $\left(23^{\circ} \mathrm{C}\right)$. The pink region delineated by the red circles represents the coacervation region (R2) where phase separation occurs; the blue area outside the blue solid circles is characterized by homogeneous solution. All solutions were prepared via dilution of a stock solution of 100 $\mathrm{g} / \mathrm{L}$ at $\mathrm{pH}$ 7. (c) Influence of $\mathrm{pH}$ on the phase boundary of coacervation. Protein solution of $11 \mathrm{~g} / \mathrm{L}$ with different initial $\mathrm{pH}$ was adjusted to different $\mathrm{NaCl}$ concentration at $23^{\circ} \mathrm{C}$. (d) Influence of temperature on the phase boundary of coacervation. Protein solution of $11 \mathrm{~g} / \mathrm{L}$ with initial $\mathrm{pH}$ of 8 was adjusted to a different $\mathrm{NaCl}$ concentration and kept at a different temperature. The phase diagrams in (b) - (d) were constructed based on the visual appearance of phase separation in the sample as well as detection of microstructures observed under the microscope. (e) Exponential decay of the aspect ratio of coalescing coacervates induced at $0.1 \mathrm{M} \mathrm{NaCl}$ of different final diameters, as given in the legend. The fits (solid curves) were used to assess the relaxation time, $\tau$, see SI. The bright field images show the coalescence of two coacervate droplets. The scale bar represents $2 \mu \mathrm{m}$. The image sequence is from Movie 1 in the SI. (f) Plot of the relaxation time $\tau$ vs the final coacervate diameter. The slope yields the inverse capillary velocity $\eta / \gamma \approx 9.69 \pm 0.53 \mathrm{~s} / \mu$ m by linear regression $\left(R^{2}=0.97, n=13\right)$.

globulins, a group of storage proteins sharing a similar structure. $^{20}$ Followed by soy glycinin, pea protein and fava bean legumin have recently been reported to form hollow structures through coacervation. ${ }^{21,22}$ However, all these studies have not offered a mechanistic view of protein-coacervate-tohollow-condensate transition. As representative of the $11 \mathrm{~S}$ globulin family, soy glycinin is a hexamer, and each of its six subunits contains one acidic and one basic polypeptide crosslinked by disulfide bonds ${ }^{23,24}$ (see also Figure S1 in the
Supporting Information, SI). This structural characteristic represents a substantial difference from coacervation-prone animal proteins featuring mostly one type of polypeptide. ${ }^{25,26}$ Here, using soy glycinin as a prototype, we aim at resolving (i) how these molecular characteristics contribute to $11 \mathrm{~S}$ globulin coacervation and the "unique" transformation into hollow condensates, (ii) what are the driving forces involved in selfassembly during coacervation and cavitation, and (iii) why the coacervates preserve their shape during the phase transition. 
(a)

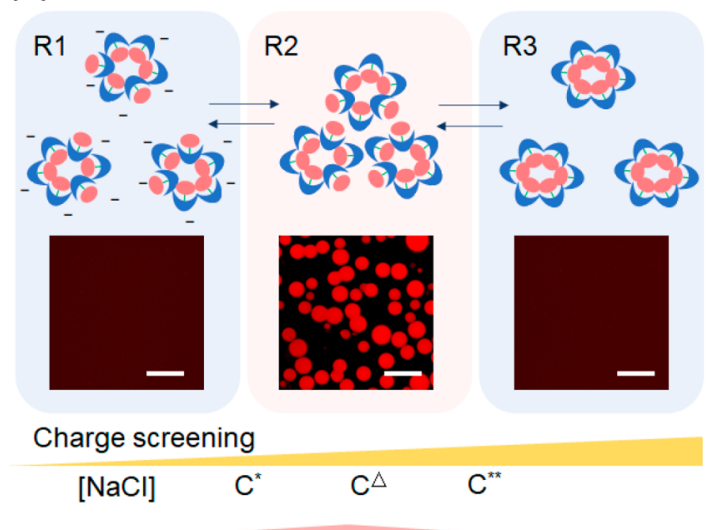

Hydrophobic interaction (b) Trimer

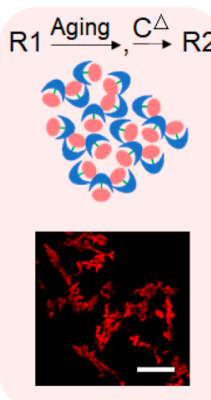

Coacervate-like flocks (c) S-S reduced

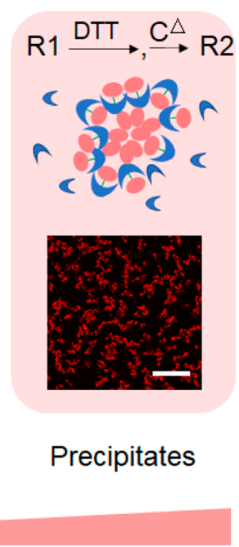

Hydrophobic interaction

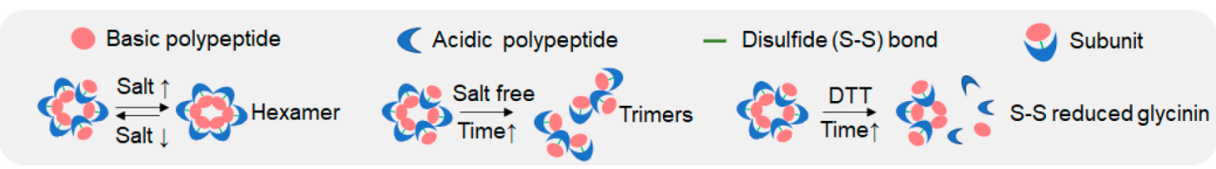

Figure 2. Schematics of the proposed molecular mechanism for soy glycinin coacervation and the structural contribution of the double-trimers stacked hexamer and the disulfide-bonded two-polypeptides subunit. The legend below illustrates the changes at single-molecule level. (a) Effect of changes of the hexamer structure with different exposures of basic and acidic polypeptides induced by altering the ionic strength. Confocal microscopy images of protein suspensions mixed with rhodamine B (which exclusively partitions into the protein-rich phase, see Figure S4) in the three characteristic regions (R1, R2, and R3 in Figure 1) are shown below. Coacervation was induced by mixing freshly prepared protein solution $(20 \mathrm{~g} / \mathrm{L}, \mathrm{pH} 7.4)$ with equal volume of $0.2 \mathrm{M} \mathrm{NaCl}$ solution to reach $\mathrm{C}^{\Delta}$ (Figure 1a). (b) Effect of partial dissociation of the protein hexamer into trimer upon aging. Protein solution of $5 \mathrm{~g} / \mathrm{L}$ was kept at $4{ }^{\circ} \mathrm{C}$ for 12 days to induce the dissociation and afterward mixed with equal volume of 0.2 $\mathrm{M} \mathrm{NaCl}$ solution to reach $C^{\Delta}$ (region R2). Instead of fluid coacervate droplets, one observes flocks, see also Figure S7a. (c) Effect of breaking disulfide bonds on coacervation. Protein solution of $10 \mathrm{~g} / \mathrm{L}$ was incubated with $20 \mathrm{mM}$ DTT for $3 \mathrm{~h}$ to reduce the disulfide bonds and subsequently mixed with equal volume of $0.2 \mathrm{M} \mathrm{NaCl}$ solution to reach $C^{\Delta}$ (region R2). The scale bars represent $20 \mu \mathrm{m}$.

To shed light on the mechanism of soy glycinin selfcoacervation, we first built the phase diagram and explore the coacervate physicochemical characteristics (Figure 1). Homogeneous soy glycinin solutions undergo sequential demixing and mixing phase transitions in response to increasing $\mathrm{NaCl}$ concentration (revealed by turbidity measurements), defining three regions in the phase space limited by lower and upper $\mathrm{NaCl}$ concentrations, $C^{*}$ and $C^{* *}$. At $[\mathrm{NaCl}]<C^{*}$ and $[\mathrm{NaCl}]>\mathrm{C}^{* *}$, the protein solution is homogeneous (regions $\mathrm{R} 1$ and R3 in Figure 1a-d). At intermediate concentrations, $C^{*}<[\mathrm{NaCl}]<C^{* *}$, coacervation takes place, leading to phase separation (region R2) with maximum turbidity at around $[\mathrm{NaCl}] \approx 0.1 \mathrm{M} \equiv \mathrm{C}^{\Delta}$ (Figure 1a). The demixing is characterized by random nucleation of spherical dense coacervates, which grow into larger ones via coalescence (see SI, Movie 1). Coalescence is slow and described by an exponential decay with relaxation time $\tau$ (Figure 1e), which depends linearly on the size of the coalescing droplets (Figure 1f), see SI, Experimental Section. The proportionality coefficient represents the inverse capillary velocity $\eta / \gamma,{ }^{27}$ where $\gamma$ is the interfacial tension driving the coalescence process and $\eta$ is the droplet viscosity that slows it down. We find $\eta / \gamma \approx 9.69 \mathrm{~s} / \mu \mathrm{m}$, which corroborates the highly viscous nature of soy glycinin coacervates (see SI, section on coalescence dynamics), also supported by negligible fluorescence recovery after photobleaching (Figure S2).

To understand the observed coacervation process, we consider the hexameric structure of soy glycinin. Every three subunits (each consisting of acidic and basic polypeptides bonded by disulfide bonds) assemble into a trimer, and two trimers stack face to face, forming the hexamer. ${ }^{23,24}$ The carboxylic ends of the acidic polypeptides are particularly divergent, also termed the hypervariable region (HVR). ${ }^{28}$ One striking feature of the HVR is the high content of negatively charged amino acids with a repeated aspartate/glutamate-rich sequence (Figure S3). ${ }^{28}$ The HVR therefore shares the characteristic of the intrinsically disordered regions (IDRs), that is, repetitive amino acid sequences with low complexity, which has been proven to be a crucial structural feature for coacervation to occur. ${ }^{29,30}$ Furthermore, the basic polypeptides exhibit a large fraction of hydrophobic amino acids, which are mostly buried inside the protein molecules, while the acidic polypeptides are hydrophilic and mostly exposed. ${ }^{31,32}$ The isoelectric point of soy glycinin is around 5.1, implying that it is negatively charged above $\mathrm{pH}$ 5.1. ${ }^{33}$ Remarkably, with increasing ionic strength, the basic polypeptides become less exposed contrary to the acidic polypeptides. ${ }^{32}$

Considering the above molecular characteristics, in Figure 2, we sketch a plausible structural interpretation of the phase behavior observed in Figure 1. Due to the strong electrostatic repulsion, at $\mathrm{pH}>7$ and $[\mathrm{NaCl}]<\mathrm{C}^{*}$, the soy glycinin solution remains homogeneous (region R1 in the phase diagram; Figure 2a). Increasing salt concentrations screens the electrostatic repulsion, allowing weak hydrophobic interactions between the exposed hydrophobic basic polypeptides and driving coacervation in region R2 (Figure 2a). This assumption is corroborated by coacervate size decrease observed in the presence of small amounts of urea (Figure S5), which is known to suppress protein-protein hydrophobic interactions. ${ }^{34,35}$ However, the coacervation was not disrupted by 1,6hexanediol, an aliphatic alcohol (Figure S5), which hinders the formation of protein condensates by perturbing weak 

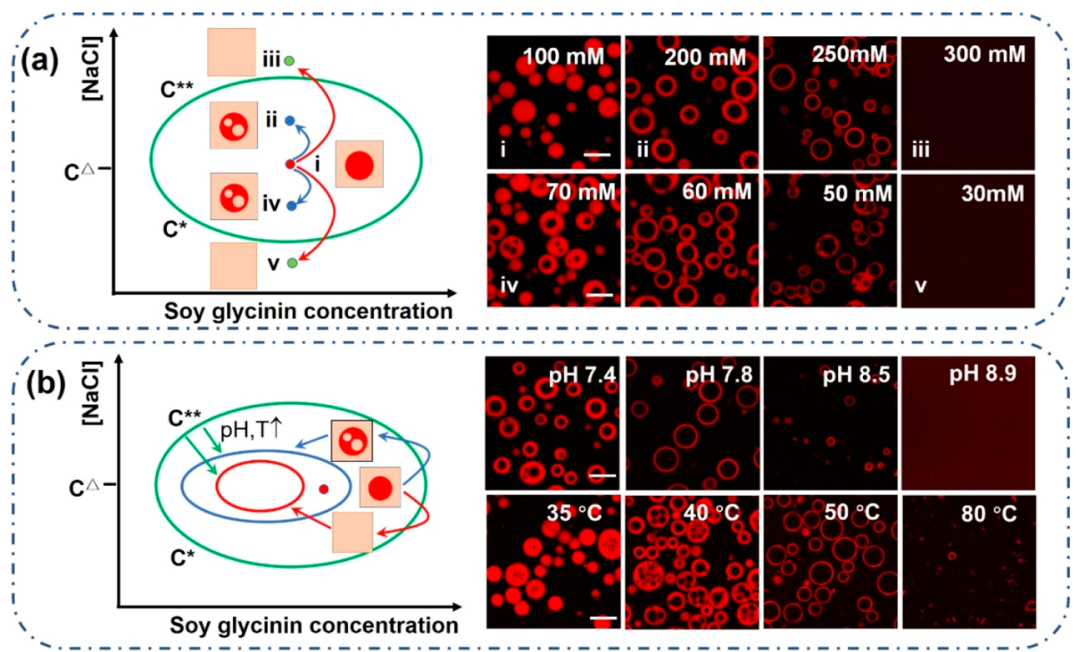

Figure 3. Phase change of soy glycinin coacervates under different solvent conditions and the formation of hollow condensates. The protein coacervates were prepared by mixing protein solution $(20 \mathrm{~g} / \mathrm{L}, \mathrm{pH} 7.4)$ with an equal volume of $0.2 \mathrm{M} \mathrm{NaCl}$ solution, yielding a final protein concentration of $10 \mathrm{~g} / \mathrm{L}$, salt concentration of $0.1 \mathrm{M}\left(\mathrm{C}^{\Delta}\right)$, and $\mathrm{pH}$ of 6.85 . The environmental condition of the suspension was then adjusted and samples were imaged immediately. (a) Influence of $[\mathrm{NaCl}]$ on the coacervate structure. Protein coacervate suspension was adjusted to different $\mathrm{NaCl}$ concentrations by mixing with equal volume of $\mathrm{NaCl}$ solution $(0-500 \mathrm{mM})$; the final $\mathrm{NaCl}$ concentration is indicated in the confocal images. The sketch on the left represents coacervate structure changes in response to shifting of the position in the phase diagram. Examples of the respective locations in the phase diagram $(\mathrm{i}-\mathrm{v})$ are illustrated with images on the right. (b) Influence of $\mathrm{pH}$ and temperature on the coacervate structure. $\mathrm{pH}$ of the coacervate suspension was increased by adding equal volume of $\mathrm{NaOH}$ solution $(0.5-1.5 \mathrm{mM})$; the final $\mathrm{pH}$ of the suspension is indicated in the images in the upper row. The coacervate suspension $(1 \mathrm{~mL})$ was heated via gentle shaking in a water bath under different temperature (indicated in the images, lower row) for $5 \mathrm{~min}$, and the microstructures were observed immediately after heating. The sketch on the left represents how increasing $\mathrm{pH}$ or temperature shrinks the phase boundary (green to blue to red ellipses) and the corresponding coacervate structure response, depending on how far it is located to (or out of) the new phase boundary. The scale bars represent $40 \mu \mathrm{m}$.

protein-protein interactions. ${ }^{36}$ This suggests that hydrophobic interactions of intermediate strength stabilize glycinin coacervates. Tentative interpretation of this stabilization at molecular lever could be sought in the high abundance of glutamine (the most frequent amino acid) and serine in soy glycinin (Figure S6a), both of which were shown to induce hardening and decreased fluidity of protein coacervates. ${ }^{37}$ Furthermore, the polyglutamine amino acid sequence (Figure $\mathrm{S} 6 \mathrm{~b})$ in the variable region might promote $\beta$-sheet structure formation, ${ }^{38}$ further contributing to the stabilization of protein droplets. $^{39}$ Above $C^{* *}$, the basic polypeptides are mostly shielded in the protein interior, exposing more acidic polypeptides, thus inhibiting coacervation due to the decreased hydrophobic interaction and yielding a homogeneous phase in region $\mathrm{R} 3$ (Figure 2a).

We also explored the effect of protein restructuring on the ability to undergo coacervation. Incubation of glycinin solutions at low salt concentration (homogeneous region $\mathrm{R} 1)$ for a long time (4-12 days) with subsequent addition of salt led to the appearance of coacervate-like flocks (Figure $2 \mathrm{~b}$, Figure S7a). This was due to the partial dissociation of the hexameric protein structure into trimers at low ionic strength $([\mathrm{NaCl}]<0.01 \mathrm{M})$ with time. ${ }^{40,41}$ The trimers expose their stacking faces making them more hydrophobic. ${ }^{23,24}$ After 12 days, the flocks could not be dissociated when $[\mathrm{NaCl}]$ was brought out of the coacervation region R2 (Figure S7b), indicating stronger hydrophobic interaction compared to those in freshly prepared coacervates. We further increased the exposure of hydrophobic basic residues by incubating glycinin with a reducing agent to break the disulfide bonds and release the hydrophilic acidic polypeptides. ${ }^{42}$ Then, upon shifting $[\mathrm{NaCl}]$ to the coacervation region $\mathrm{R} 2$, we observe the transformation of the spherical coacervates to coacervate-like flocks and later on precipitates (Figure 2c and Figure S7c). These precipitates also persist at salt concentrations out of the coacervation region (Figure S7d).

Considering all of the above experiments, we conclude that glycinin coacervation is driven by charge screening of the acidic polypeptides, which promotes weak hydrophobic interactions between the exposed basic polypeptides as typically observed for other proteins. ${ }^{26,43}$ The enhanced stability of glycinin droplets against 1,6-hexanediol evidences the presence of even stronger hydrophobic interactions. Importantly, the hexameric structure of glycinin combined with the effect of disulfide bonding shields the basic polypeptides ensuring hydrophobic interaction that is weak enough to induce coacervation rather than flocking or precipitation. Contribution of charge screening to coacervation is further evidenced by the influence of $\mathrm{pH}$ on the phase boundary (Figure 1c). Raising the $\mathrm{pH}$ above 7 shrinks the phase-separation region $\mathrm{R} 2$. At $\mathrm{pH}>8.7$, coacervation is completely suppressed because of the increased negative surface charge of glycinin, ${ }^{33}$ which weakens the screening effect of salt. Below the isoelectric point $(\sim \mathrm{pH} 5.1)$, glycinin becomes positively charged and the electrostatic repulsion increases with decreasing $\mathrm{pH}^{33}$ The coacervation is therefore suppressed when $\mathrm{pH}$ is below 3.5 (Figure S8). Similarly, increasing temperature from 4 to $50{ }^{\circ} \mathrm{C}$, as shown in Figure $1 \mathrm{~d}$, also shrinks the region $\mathrm{R} 2$. At $\mathrm{T}>55{ }^{\circ} \mathrm{C}$, coacervation is completely suppressed because of the enhanced hydrophobic interaction between soy glycinin molecules that favors the thermally induced irreversible aggregation. ${ }^{44}$

Considering that ionic strength, $\mathrm{pH}$, and temperature significantly influence the coacervation (Figure 1), we hypothesized that soy glycinin coacervates should show responsive structural changes upon modifying these environ- 

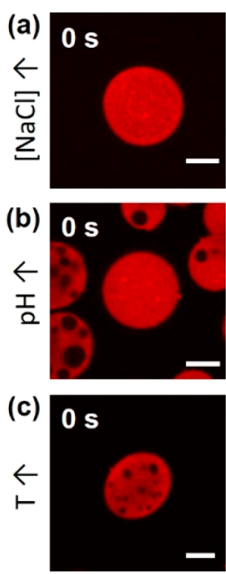
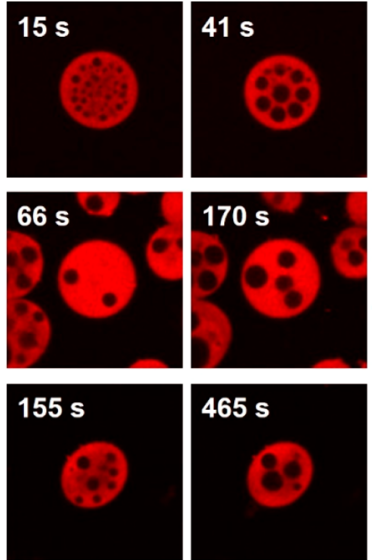

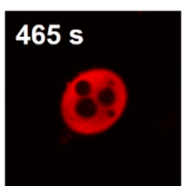

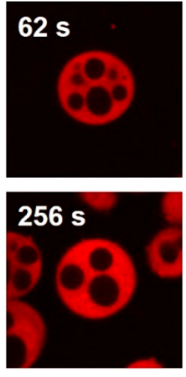

$675 \mathrm{~s}$

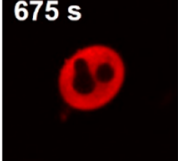

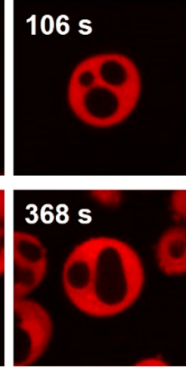

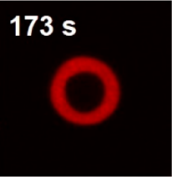

$510 \mathrm{~s}$
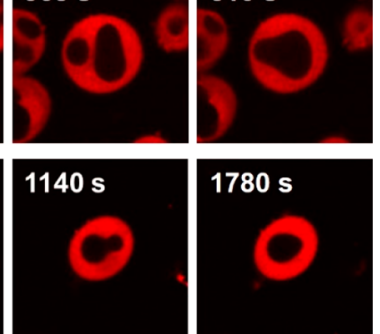

$1780 \mathrm{~s}$

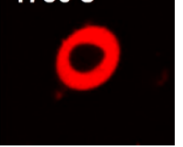

Figure 4. Formation of hollow condensates (cavitation) upon salt, $\mathrm{pH}$ or temperature increase. Time-lapse confocal microscopy cross-section images showing cavity formation in the protein condensates under different conditions. Protein coacervates were prepared by mixing protein solution $(20 \mathrm{~g} / \mathrm{L}, \mathrm{pH} 7.4)$ with equal volume of $0.2 \mathrm{M} \mathrm{NaCl}$ solution at $23{ }^{\circ} \mathrm{C}$, giving a final protein concentration of $10 \mathrm{~g} / \mathrm{L}, \mathrm{NaCl}$ concentration of $0.1 \mathrm{M}$ and $\mathrm{pH}$ of 6.85 . Ionic strength, $\mathrm{pH}$, and temperature of the suspensions were then adjusted and the corresponding protein-rich phase evolution was visualized by rhodamine B fluorescence shortly after preparation (time 0 corresponds to $\sim 30 \mathrm{~s}$ after salt, $\mathrm{pH}$, or temperature adjustment). (a) The salt concentration of the coacervate suspension was increased from 0.1 to $0.175 \mathrm{M}$. The image sequence corresponds to Movie 2 in the SI. (b) The $\mathrm{pH}$ of the coacervate suspension was increased from to 6.85 to 8.2; Movie 3 in the SI. (c) The coacervate suspension was incubated at $40{ }^{\circ} \mathrm{C}$; Movie 4 in the SI. The scale bars represent $10 \mu \mathrm{m}$.

mental conditions. Previous research only reported heatinduced transition from coacervates to hollow condensates. Here, we find that any factor that brings the coacervation system toward the binodals can induce such transition. We consider coacervates formed at $[\mathrm{NaCl}]=C^{\Delta} \equiv 0.1 \mathrm{M}$. Upon shifting $[\mathrm{NaCl}]$ toward the binodals $\left(C^{*} / C^{* *}\right)$, the coacervates transform into hollow condensates with gradually decreasing fraction of the dense phase (Figure $3 \mathrm{a}$ ) in accordance with the turbidity measurement (Figure 1a). Beyond the phase boundary, the coacervates disperse into a homogeneous phase (Figure 3a). Increasing $\mathrm{pH}$ or temperature results in shrinking the coacervation region (R2 in Figure $1 \mathrm{c}, \mathrm{d})$, thus, bringing the phase boundary closer to the location of our system in the phase diagram (Figure $3 \mathrm{~b}$ ). The condensates again adjust their phase state by forming a protein-poor phase inside (and transforming the droplets into hollow condensates) or dissolve when the system is beyond the new binodal (Figure 3b). Decreasing protein concentration by dilution could also lead to cavity formation, which is more pronounced when the system is near the coacervation boundary (Figure S9).

We further investigated the process of cavitation as a first step to understand the mechanism of hollow-condensate formation. We prepared protein coacervates at $[\mathrm{NaCl}]=C^{\Delta} \equiv$ $0.1 \mathrm{M}$, and subsequently increased the salt concentration to $0.175 \mathrm{M}$ (i.e., still in region $\mathrm{R} 2$ of the phase diagram, Figure 1b). Many small cavities first formed inside the condensates and then merged into a larger one typically within minutes (Figure $4 \mathrm{a}$ and Movie 2). Interestingly, cavity formation induced by increasing $\mathrm{pH}$ (Figure $4 \mathrm{~b}$ and Movie 3 ) or temperature (Figure $4 \mathrm{c}$ and Movie 4 ) follows the same process as that induced by salt. A similar coacervate-to-hollow structure transition has been described for pea protein isolate ${ }^{21}$ as well as for RNA-protein (complex) coacervates in vivo and in vitro, ${ }^{45-47}$ although the nature and behavior of the latter as complex coacervates are quite different from those of soy glycinin (simple) coacervates. It should be noted that, for conditions of $[\mathrm{NaCl}]<C^{\Delta}$, small irregularly shaped coacervates were often observed (Figure S10). A few seconds of heating would transform them into microdomains with smooth boundaries, followed by the nucleation and growth of protein-poor phase in their interior (Figure S10). This intermediate morphological transition clarifies why irregular coacervate clusters can also form hollow structures upon heating as observed earlier. ${ }^{48}$

The mechanism of hollow-condensate formation is partially related to the interplay of charge screening and hydrophobic interactions. Upon shifting $[\mathrm{NaCl}]$ toward $C^{* *}$, the hydrophobic interaction stabilizing the coacervates decreases as more acidic polypeptides are exposed. Both shifting $[\mathrm{NaCl}]$ toward $C^{*}$ and increasing $\mathrm{pH}$ enhance the electrostatic repulsion, which weaken the salt screening effects. Increasing temperature leads to a stronger hydrophobic interaction that is unfavorable for coacervation. These changes in the molecular interactions lead to coacervate dissociation, but they do not explain why the protein-poor phase forms in the interior instead of simply shrinking the condensate in size. We speculated that enhanced surface interaction or organization stabilizes the observed shapes. We therefore probed the condensates for structural order (as observed for RNAprotein complexes ${ }^{45}$ ) using polarization microscopy (Figure 5a). Both coacervates and hollow condensates display strong birefringence at their surface, suggesting surface organization of the protein-rich phase. This liquid-crystalline-like order persists during the transformation into hollow condensates. Interestingly, the inner surface of the hollow condensates also shows birefringence, indicating order. We propose that, at these interfaces, the protein orients so that the more hydrophilic acidic polypeptides extend toward the protein-depleted phase, while the more hydrophobic exposed basic polypeptides extend toward the protein-dense phase, thus, leading to ordered packing (Figure 6). This molecular reorientation presumably strengthens the hydrophobic interactions at these organized surfaces, which is supported by the observation that, in the presence of urea, coacervate or hollow-condensate dissolution initiates from the dense phase to the surface (Figure S11). The surface order and increased protein 


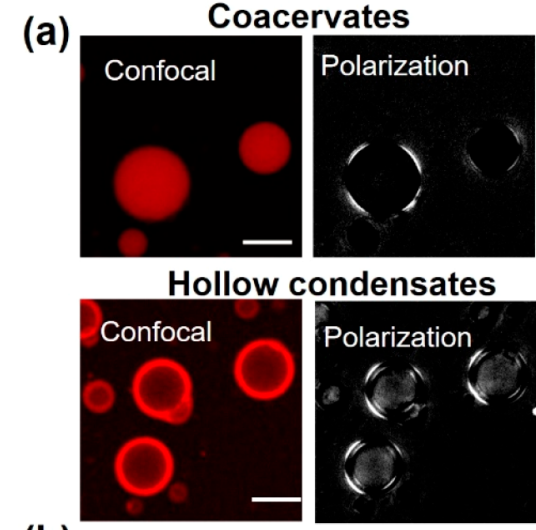

(b)

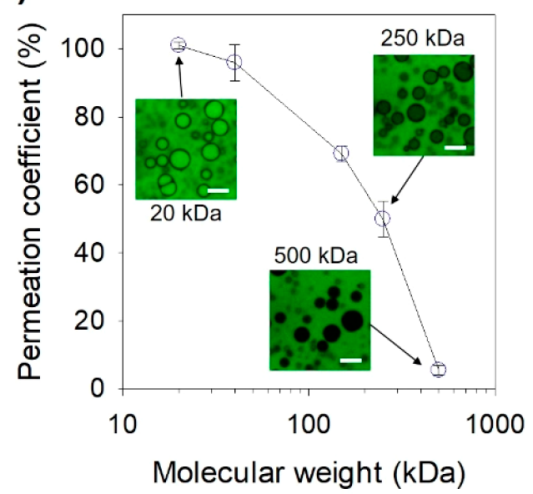

(c)

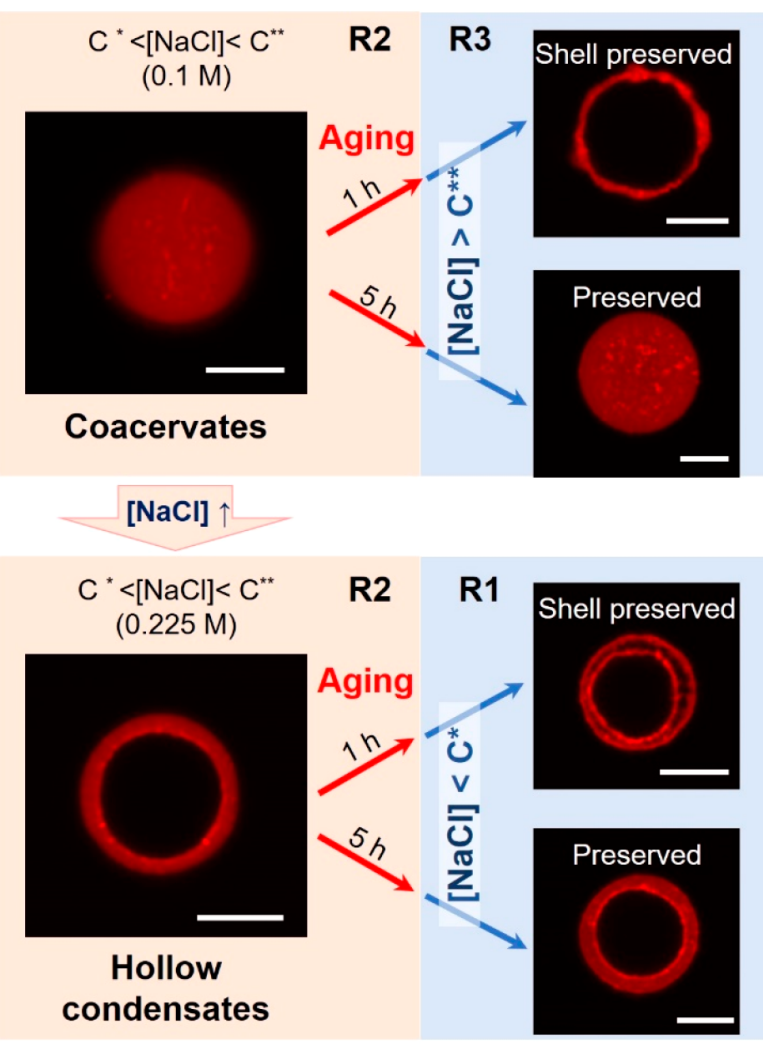

Figure 5. Structural order at coacervate and hollow-condensate surfaces, mesh size of surface and dense phase and aging conditions for preserved topology beyond the coacervation region R2. Protein coacervates were prepared by mixing soy glycinin solution (20 g/L, $\mathrm{pH} 7.4)$ with equal volume of $0.2 \mathrm{M} \mathrm{NaCl}$ solution at $23{ }^{\circ} \mathrm{C}$ to yield a protein suspension with protein concentration of $10 \mathrm{~g} / \mathrm{L}$ and $\mathrm{NaCl}$ concentration of $0.1 \mathrm{M}$. Hollow condensates were induced by increasing the ionic strength to $0.225 \mathrm{M}$. (a) Optical images of coacervates and hollow condensates with cross-polarizing light show birefringence, indicating molecular ordering at the outer and cavity surfaces. Corresponding fluorescence micrographs are also shown. The scale bars represent $20 \mu \mathrm{m}$. (b) Permeation coefficient of FITC-labeled dextran as a function of molecular weight (see Figure S14 for details on the measurement). The measurements were performed on hollow condensates $(n=5)$. The error bars indicate standard deviation. The inserts show representative images of the permeation of dextran in the hollow coacervates. The scale bars represent $40 \mu \mathrm{m}$. (c) Influence of aging on the stability of coacervates and hollow condensates upon shifts beyond the phase boundary of the coacervation region R2; see more details in Figure S15. Aging was done at $23{ }^{\circ} \mathrm{C}$ under mild stirring and for different periods (indicated above the red arrows), after which the salt concentration of the suspension was shifted to $0.03 \mathrm{M}$, which falls into the homogeneity region $\mathrm{R} 1$, that is, $[\mathrm{NaCl}]<\mathrm{C}^{*}$, or $0.3 \mathrm{M}$, which falls into homogeneity region $\mathrm{R} 3$, that is, $[\mathrm{NaCl}]>C^{* *}$. The scale bars represent $10 \mu \mathrm{m}$.

interactions could contribute to the long stability of the hollow condensates (Figure S12), as discussed below.

Considering that part of the glycinin molecules in the coacervate interior become mobile and are released out of the coacervates upon cavitation, the dense phase and ordered surface should have a mesh size that allows the diffusion of free glycinin. We probed this mesh size using FITC-labeled dextran. Dextran, with a $M_{\mathrm{w}}>4 \mathrm{kDa}$, was practically excluded from the protein-rich phase of the coacervates or the hollow condensates (Figures S13 and S14). Upon cavity formation, low-molecular-weight dextran $(\leq 40 \mathrm{kDa})$ could diffuse through the capsule-like dense phase, while $500 \mathrm{kDa}$ dextran could not permeate (Figures 5b and S14). Presumably, the ordered surface acts as a size-dependent filter with a mesh size of at least 3-5 nm, as defined by the radius of gyration of 20$40 \mathrm{kDa}$ dextran, ${ }^{49}$ that would therefore allow free glycinin, which has a radius around $4 \mathrm{~nm}^{50,51}$ to leak out rather than concentrating in the layer of protein-dense phase.

Soy proteins tend to aggregate and gel even at low temperature, promoted by increasing protein concentration. ${ }^{52,53}$ Considering that the condensates represent a highly dense protein phase, it is plausible that protein-protein interactions are enhanced with time. To resolve the influence of aging and probe the stability of the resulting structures, we aged protein coacervates at $23{ }^{\circ} \mathrm{C}$ for different periods and subsequently examined their stability by shifting the salt concentration out of the coacervation region $\mathrm{R} 2$ and into the homogeneity regions ( $\mathrm{R} 1 / \mathrm{R} 3)$. We were, thus, able to resolve aging conditions that lead to preserving the structures. After a short aging time, the coacervates could still be dissociated irrespective of the $[\mathrm{NaCl}]$ shift direction (Figure S15a). In samples aged for $1 \mathrm{~h}$, the coacervate surface retained its topology (Figure 5c, upper panel) in region R3, suggesting stronger protein-protein interactions resulting from the surface order evidenced by the polarization microscopy images (Figure 5a). At longer times $(>5 \mathrm{~h})$, the whole coacervate appeared preserved (Figure 5c, upper panel).

Aging also strengthened the stability of hollow condensates. Short aging times $(\sim 10 \mathrm{~min})$ led to thinning of the proteindense phase or partial dissolution (Figure S15b). After aging for $1 \mathrm{~h}$, the dense phase was released, leaving the outer and inner surfaces of the hollow condensates as concentric shells in region R1 (Figure 5c, lower panel, and Figure S15b). This confirms that protein molecules at the inner surface of the 


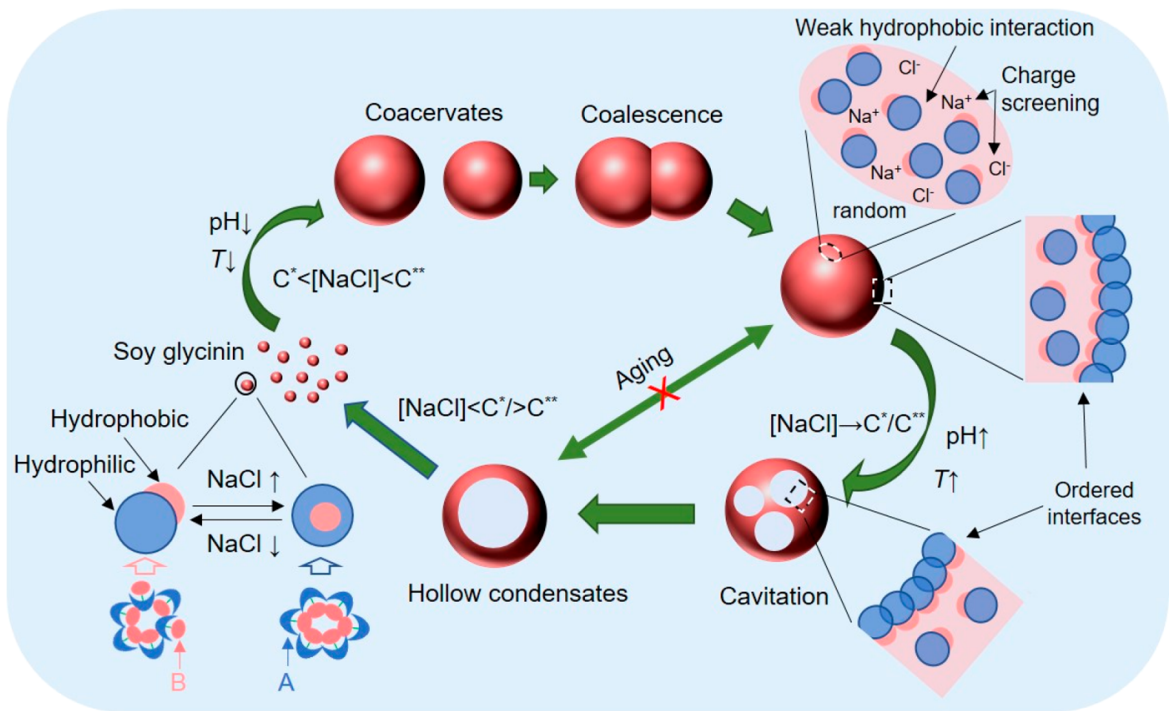

Figure 6. Schematic illustration of the mechanisms of the self-coacervation of soy glycinin, the transformation from coacervates to hollow condensates and their surface organization. The hexameric structure of glycinin contributes to these phase changes via the interplay of the basic (B, hydrophobic) and acidic (A, hydrophilic) polypeptides, which modulate the protein-protein interactions upon environmental condition changes, as shown in Figure 2. The structure of the hexamer was simplified with one hydrophilic part (blue), representing the exposed acidic polypeptides and one hydrophobic part (pink), representing the exposed basic polypeptides. Homogeneous solutions of the protein (left) undergo coacervation in a specific range of salt concentration $\left(\mathrm{C}^{*}<[\mathrm{NaCl}]<\mathrm{C}^{* *}\right), \mathrm{pH}$, and temperature, which is driven by charge screening of acidic polypeptides and the hydrophobic interaction between exposed basic polypeptides. The coacervates are fluid and can grow via coalescence. Upon changes in the external conditions bringing the system closer to the binodal ( $\mathrm{C}^{*}$ or $\mathrm{C}^{* *}$ ) or upon increasing temperature or $\mathrm{pH}$, the coacervates develop cavities of the protein-poor phase, which transforms the droplets into hollow condensates. This process is irreversible and is hindered by aging. The amphiphilic glycinin molecules orient at condensate interface, rendering surface order that strengthens intermolecular interactions stabilizing the coacervate shape. In fresh solutions, the dense phase dissolves once the external conditions shift the system out of the region of phase coexistence, while in aged ones, the structures are preserved.

hollow condensates assemble similar to those at the outer surface, in accordance with the polarization microscopy observations. Overall, aging led to the formation of stabilized shells offering a facile alternative pathway for microcapsule production. The enhanced stability with aging is presumably due to the large abundance of glutamine and serine (Figure S6a), which promotes the hardening of coacervates in a timedependent manner. ${ }^{37}$ The polyglutamine sequence might also contribute to the stabilization of protein droplets by forming higher-order structure (Figure S6b). ${ }^{38}$ Overall, the hydrophobic interactions are strengthened with aging (Figure S16).

The proposed molecular mechanism behind coacervate formation, transformation into hollow-condensates, and shape stability is summarized in Figure 6. Remarkably, aging enhances the protein-protein interaction, transforming the coacervates or hollow condensates into microcapsules with a morphology stable to solvent condition changes. This research provides essential knowledge and approaches for investigating the coacervation of other plant seed $11 \mathrm{~S}$ globulins. Furthermore, it paves the way toward designing novel microstructures such as semipermeable responsive microcapsules as well as further understanding of the accumulation and dissociation of the protein condensates in plant seed cells. $^{54,55}$

\section{ASSOCIATED CONTENT}

\section{(s) Supporting Information}

The Supporting Information is available free of charge at https://pubs.acs.org/doi/10.1021/acsmacrolett.0c00709.

Details for the materials and experimental methods, supporting figures, and movie captions (PDF)
Movie 1: Coalescence dynamics of protein coacervates (MP4)

Movie 2: Transition from coacervates to hollow condensates induced by increasing ionic strength (AVI)

Movie 3: Transition from coacervates to hollow condensates induced by increasing $\mathrm{pH}$ (AVI)

Movie 4: Transition from coacervates to hollow condensates induced by increasing temperature (AVI)

\section{AUTHOR INFORMATION}

\section{Corresponding Authors}

Nannan Chen - Guangdong Saskatchewan Oilseed Joint Laboratory, Department of Food Science and Engineering, Jinan University, 510632 Guangzhou, China; Max Planck Institute of Colloids and Interfaces, Department of Theory and Bio-Systems, 14424 Potsdam, Germany;

Email: nannanchen@jnu.edu.cn

Rumiana Dimova - Max Planck Institute of Colloids and Interfaces, Department of Theory and Bio-Systems, 14424 Potsdam, Germany; 이이.org/0000-0002-3872-8502; Email: rumiana.dimova@mpikg.mpg.de

\section{Authors}

Ziliang Zhao - Max Planck Institute of Colloids and Interfaces, Department of Theory and Bio-Systems, 14424 Potsdam, Germany; 이이.org/0000-0002-8723-7312

Yong Wang - Guangdong Saskatchewan Oilseed Joint Laboratory, Department of Food Science and Engineering, Jinan University, 510632 Guangzhou, China; 10 orcid.org/ 0000-0001-7547-1542 
Complete contact information is available at:

https://pubs.acs.org/10.1021/acsmacrolett.0c00709

\section{Notes}

The authors declare no competing financial interest.

\section{ACKNOWLEDGMENTS}

N.C. acknowledges support from China Postdoctoral Innovation Talent Support Program (BX20190145), China Postdoctoral Science Foundation (2019M663387), and Natural Science Foundation of Guangdong Province (2019A1515011176). Z.Z. and R.D. thank the MaxSynBio consortium, which is jointly funded by the Federal Ministry of Education and Research (BMBF) of Germany and the Max Planck Society (MPG). We thank N. Yandrapalli for assistance with polarization microscopy and Jun Zhu for the SDS-PAGE.

\section{REFERENCES}

(1) Alberti, S.; Gladfelter, A.; Mittag, T. Considerations and Challenges in Studying Liquid-Liquid Phase Separation and Biomolecular Condensates. Cell 2019, 176 (3), 419-434.

(2) Mohanty, B.; Bohidar, H. B. Systematic of Alcohol-Induced Simple coacervation in Aqueous Gelatin Solutions. Biomacromolecules 2003, 4, 1080-1086.

(3) Croguennec, T.; Tavares, G. M.; Bouhallab, S. Heteroprotein complex coacervation: A generic process. Adv. Colloid Interface Sci. 2017, 239, 115-126.

(4) Mitrea, D. M.; Kriwacki, R. W. Phase separation in biology; functional organization of a higher order. Cell Commun. Signaling 2016, 14, 1.

(5) Banani, S. F.; Lee, H. O.; Hyman, A. A.; Rosen, M. K. Biomolecular condensates: organizers of cellular biochemistry. Nat. Rev. Mol. Cell Biol. 2017, 18 (5), 285-298.

(6) Mohammadi, P.; Aranko, A. S.; Lemetti, L.; Cenev, Z.; Zhou, Q.; Virtanen, S.; Landowski, C. P.; Penttilä, M.; Fischer, W. J.; Wagermaier, W.; Linder, M. B. Phase transitions as intermediate steps in the formation of molecularly engineered protein fibers. Commun. Biol. 2018, 1 (1), 86.

(7) Kim, S.; Yoo, H. Y.; Huang, J.; Lee, Y.; Park, S.; Park, Y.; Jin, S.; Jung, Y. M.; Zeng, H.; Hwang, D. S.; Jho, Y. Salt Triggers the Simple Coacervation of an Underwater Adhesive When Cations Meet Aromatic pi Electrons in Seawater. ACS Nano 2017, 11 (7), 67646772.

(8) Black, K. A.; Priftis, D.; Perry, S. L.; Yip, J.; Byun, W. Y.; Tirrell, M. Protein Encapsulation via Polypeptide Complex Coacervation. ACS Macro Lett. 2014, 3 (10), 1088-1091.

(9) Nishinari, K.; Fang, Y.; Guo, S.; Phillips, G. O. Soy proteins: A review on composition, aggregation and emulsification. Food Hydrocolloids 2014, 39, 301-318.

(10) Renkema, J. M. S.; Gruppen, H.; Van Vliet, T. Influence of pH and ionic strength on heat-induced formation and rheological properties of soy protein gels in relation to denaturation and their protein compositions. J. Agric. Food Chem. 2002, 50, 6064-6071.

(11) Mo, X.; Zhong, Z.; Wang, D.; Sun, X. Soybean Glycinin Subunits: Characterization of Physicochemical and Adhesion Properties. J. Agric. Food Chem. 2006, 54, 7589-7593.

(12) Chen, N.; Zhao, M.; Nicolai, T.; Chassenieux, C. Exploiting Salt Induced Microphase Separation To Form Soy Protein Microcapsules or Microgels in Aqueous Solution. Biomacromolecules 2017, 18 (7), 2064-2072.

(13) Chen, N.; Zhang, J.; Mei, L.; Wang, Q. Ionic Strength and $\mathrm{pH}$ Responsive Permeability of Soy Glycinin Microcapsules. Langmuir 2018, 34 (33), 9711-9718.

(14) Nesterenko, A.; Alric, I.; Silvestre, F.; Durrieu, V. Vegetable proteins in microencapsulation: A review of recent interventions and their effectiveness. Ind. Crops Prod. 2013, 42, 469-479.
(15) Can Karaca, A.; Low, N. H.; Nickerson, M. T. Potential use of plant proteins in the microencapsulation of lipophilic materials in foods. Trends Food Sci. Technol. 2015, 42 (1), 5-12.

(16) Lazko, J.; Popineau, Y.; Legrand, J. Soy glycinin microcapsules by simple coacervation method. Colloids Surf., B 2004, 37 (1-2), 18.

(17) Li, X.; Erni, P.; van der Gucht, J.; de Vries, R. Encapsulation Using Plant Proteins: Thermodynamics and Kinetics of Wetting for Simple Zein Coacervates. ACS Appl. Mater. Interfaces 2020, 12 (13), $15802-15809$.

(18) Zhao, H.; Guo, M.; Ma, X.; Ding, T.; Liu, D. Microstructure and permeability of hollow microcapsules produced from faba bean 11s protein. Food Hydrocolloids 2021, 112, 106292.

(19) Tong, W.; Song, X.; Gao, C. Layer-by-layer assembly of microcapsules and their biomedical applications. Chem. Soc. Rev. 2012, 41, 6103-6124.

(20) Tandang-Silvas, M. R.; Fukuda, T.; Fukuda, C.; Prak, K.; Cabanos, C.; Kimura, A.; Itoh, T.; Mikami, B.; Utsumi, S.; Maruyama, $\mathrm{N}$. Conservation and divergence on plant seed 11S globulins based on crystal structures. Biochim. Biophys. Acta, Proteins Proteomics 2010, 1804 (7), 1432-1442.

(21) Cochereau, R.; Nicolai, T.; Chassenieux, C.; Silva, J. V. C. Mechanism of the spontaneous formation of plant protein microcapsules in aqueous solution. Colloids Surf., A 2019, 562, 213-219.

(22) Zhao, H.; Zhou, X.; Wang, J.; Ma, X.; Guo, M.; Liu, D. Heatinduced hollow microcapsule formation using fava bean legumin. Food Hydrocolloids 2021, 112, 106207.

(23) Adachi, M.; Kanamori, J.; Masuda, T.; Yagasaki, K.; Kitamura, K.; Mikami, B.; Utsumi, S. Crystal structure of soybean $11 \mathrm{~S}$ globulin: Glycinin A3B4 homohexamer. Proc. Natl. Acad. Sci. U. S. A. 2003, 100, $7395-7400$.

(24) Adachi, M.; Takenaka, Y.; Gidamis, A. B.; Mikami, B.; Utsumi, $\mathrm{S}$. Crystal structure of soybean proglycinin $\mathrm{AlaB} 1 \mathrm{~b}$ homotrimer. $J$. Mol. Biol. 2001, 305 (2), 291-305.

(25) Gabryelczyk, B.; Cai, H.; Shi, X.; Sun, Y.; Swinkels, P. J. M.; Salentinig, S.; Pervushin, K.; Miserez, A. Hydrogen bond guidance and aromatic stacking drive liquid-liquid phase separation of intrinsically disordered histidine-rich peptides. Nat. Commun. 2019, 10 (1), 5465.

(26) Wei, W.; Tan, Y.; Martinez Rodriguez, N. R.; Yu, J.; Israelachvili, J. N.; Waite, J. H. A mussel-derived one component adhesive coacervate. Acta Biomater. 2014, 10 (4), 1663-1670.

(27) Eggers, J.; Lister, J. R.; Stone, H. A. Coalescence of liquid drops. J. Fluid Mech. 1999, 401, 293-310.

(28) Nielsen, N. C.; Dickinson, C. D.; Cho, T.-J.; Thanh, V. H.; Scallon, B. J.; Fischer, R. L.; Sims, T. L.; Drews, C. N.; Goldberg, R. B. Characterization of the glycinin gene family in soybean. Plant Cell 1989, 1, 313-328.

(29) Majumdar, A.; Dogra, P.; Maity, S.; Mukhopadhyay, S. LiquidLiquid Phase Separation Is Driven by Large-Scale Conformational Unwinding and Fluctuations of Intrinsically Disordered Protein Molecules. J. Phys. Chem. Lett. 2019, 10 (14), 3929-3936.

(30) Schuster, B. S.; Dignon, G. L.; Tang, W. S.; Kelley, F. M.; Ranganath, A. K.; Jahnke, C. N.; Simpkins, A. G.; Regy, R. M.; Hammer, D. A.; Good, M. C.; Mittal, J. Identifying sequence perturbations to an intrinsically disordered protein that determine its phase-separation behavior. Proc. Natl. Acad. Sci. U. S. A. 2020, 117 (21), 11421-11431.

(31) Yuan, D.-B.; Yang, X.-Q.; Tang, C.-H.; Zheng, Z.-X.; Wei, M.; Ahmad, I.; Yin, S.-W. Physicochemical and functional properties of acidic and basic polypeptides of soy glycinin. Food Res. Int. 2009, 42 (5-6), 700-706.

(32) Lakemond, C. M. M.; de Jongh, H. H. J.; Hessing, M.; Gruppen, H.; Voragen, A. G. J. Soy glycinin: Influence of $\mathrm{pH}$ and ionic strength on solubility and molecular structure at ambient temperatures. J. Agric. Food Chem. 2000, 48, 1985-1990.

(33) Yuan, Y.; Wan, Z.-L.; Yang, X.-Q.; Yin, S.-W. Associative interactions between chitosan and soy protein fractions: Effects of $\mathrm{pH}$, 
mixing ratio, heat treatment and ionic strength. Food Res. Int. 2014, $55,207-214$

(34) Kim, J. R.; Muresan, A.; Lee, K. Y.; Murphy, R. M. Urea modulation of beta-amyloid fibril growth: experimental studies and kinetic models. Protein Sci. 2004, 13 (11), 2888-2898.

(35) Li, H. R.; Chen, T. C.; Hsiao, C. L.; Shi, L.; Chou, C. Y.; Huang, J. R. The physical forces mediating self-association and phaseseparation in the C-terminal domain of TDP-43. Biochim. Biophys. Acta, Proteins Proteomics 2018, 1866 (2), 214-223.

(36) Ambadipudi, S.; Reddy, J. G.; Biernat, J.; Mandelkow, E.; Zweckstetter, M. Residue-specific identification of phase separation hot spots of Alzheimer's-related protein tau. Chem. Sci. 2019, 10 (26), 6503-6507.

(37) Wang, J.; Choi, J. M.; Holehouse, A. S.; Lee, H. O.; Zhang, X.; Jahnel, M.; Maharana, S.; Lemaitre, R.; Pozniakovsky, A.; Drechsel, D.; Poser, I.; Pappu, R. V.; Alberti, S.; Hyman, A. A. A Molecular Grammar Governing the Driving Forces for Phase Separation of Prion-like RNA Binding Proteins. Cell 2018, 174 (3), 688-699.

(38) Darnell, G.; Orgel, J. P.; Pahl, R.; Meredith, S. C. Flanking polyproline sequences inhibit beta-sheet structure in polyglutamine segments by inducing PPII-like helix structure. J. Mol. Biol. 2007, 374 (3), 688-704.

(39) Lin, Y.; Protter, D. S.; Rosen, M. K.; Parker, R. Formation and Maturation of Phase-Separated Liquid Droplets by RNA-Binding Proteins. Mol. Cell 2015, 60 (2), 208-219.

(40) Wolf, W. J.; Briggs, D. R. Studies on the cold insoluble fraction of the water extractable soybean proteins. II. Factors influencing conformation changes in the $11 \mathrm{~S}$ Component. Arch. Biochem. Biophys. 1958, 76, 377-393.

(41) Wolf, W. J.; Rackis, J. J.; Smith, A. K.; Sasame, H. A.; Babcock, G. E. Behavior of the $11 \mathrm{~S}$ Protein of soybeans in acid solutions. I. Effects of $\mathrm{pH}$, ionic strength and time on ultracentrifugal and optical rotatory properties. J. Am. Chem. Soc. 1958, 80, 5730-5735.

(42) Wolf, W. J. Sulfhydryl content of glycinin: effect of reducing agents. J. Agric. Food Chem. 1993, 41, 168-176.

(43) Cai, H.; Gabryelczyk, B.; Manimekalai, M. S. S.; Grüber, G.; Salentinig, S.; Miserez, A. Self-coacervation of modular squid beak proteins - a comparative study. Soft Matter 2017, 13 (42), 77407752.

(44) Guo, J.; Yang, X. Q.; He, X. T.; Wu, N. N.; Wang, J. M.; Gu, W.; Zhang, Y. Y. Limited aggregation behavior of beta-conglycinin and its terminating effect on glycinin aggregation during heating at $\mathrm{pH}$ 7.0. J. Agric. Food Chem. 2012, 60 (14), 3782-3791.

(45) Alshareedah, I.; Moosa, M. M.; Raju, M.; Potoyan, D. A.; Banerjee, P. R. Phase transition of RNA-protein complexes into ordered hollow condensates. Proc. Natl. Acad. Sci. U. S. A. 2020, 117, $15650-15658$.

(46) Kistler, K. E.; Trcek, T.; Hurd, T. R.; Chen, R.; Liang, F.-X.; Sall, J.; Kato, M.; Lehmann, R. Phase transitioned nuclear Oskar promotes cell division of Drosophila primordial germ cells. eLife 2018, 7, e37949.

(47) Banerjee, P. R.; Milin, A. N.; Moosa, M. M.; Onuchic, P. L.; Deniz, A. A. Reentrant Phase Transition Drives Dynamic Substructure Formation in Ribonucleoprotein Droplets. Angew. Chem., Int. Ed. 2017, 56 (38), 11354-11359.

(48) Zhao, H. H.; Guo, M. M.; Ding, T.; Ye, X. Q.; Liu, D. H. Exploring the mechanism of hollow microcapsule formation by selfassembly of soy $11 \mathrm{~s}$ protein upon heating. Food Hydrocolloids 2020, $108,105379$.

(49) Andrieux, K.; Lesieur, P.; Lesieur, S.; Ollivon, M.; GrabielleMadelmont, C. c. Characterization of Fluorescein IsothiocyanateDextrans Used in Vesicle Permeability Studies. Anal. Chem. 2002, 74, 5217-5226.

(50) Badley, A. R.; Atkinson, D.; Hauser, H.; Oldani, D.; Green, J. P.; Stubbs, J. M. The structure, physical and chemical properteis of soy bean protein glycinin. Biochim. Biophys. Acta, Protein Struct. 1975, 412, 214-228.

(51) Sokolova, A.; Kealley, C. S.; Hanley, T.; Rekas, A.; Gilbert, E. P. Small-angle X-ray scattering study of the effect of $\mathrm{pH}$ and salts on $11 \mathrm{~S}$ soy glycinin in the freeze-dried powder and solution states. J. Agric. Food Chem. 2010, 58 (2), 967-974.

(52) Chen, N.; Zhao, M.; Chassenieux, C.; Nicolai, T. The effect of adding $\mathrm{NaCl}$ on thermal aggregation and gelation of soy protein isolate. Food Hydrocolloids 2017, 70, 88-95.

(53) Chen, N.; Nicolai, T.; Chassenieux, C.; Wang, Y. pH and ionic strength responsive core-shell protein microgels fabricated via simple coacervation of soy globulins. Food Hydrocolloids 2020, 105, 105853.

(54) Dawidowicz-Grzegorzewska, A. Degradation of Protein and Lipid Bodies during Dormancy Removal in Apple Seeds. J. Plant Physiol. 1989, 135 (1), 43-51.

(55) Mori, T.; Maruyama, N.; Nishizawa, K.; Higasa, T.; Yagasaki, K.; Ishimoto, M.; Utsumi, S. The composition of newly synthesized proteins in the endoplasmic reticulum determines the transport pathways of soybean seed storage proteins. Plant J. 2004, 40 (2), 238-249. 